\title{
Whirl Plus Tilt
}

\author{
T. K. Fowler
}

October 12, 1994

This is an informal report intended primarily for internal or limited external distribution. The opinions and conclusions stated are those of the author and may or may not be those of the Laboratory.

Work performed under the auspices of the U.S. Department of Energy by the Lawrence Livermore National Laboratory under Contract W-7405-Eng-48. 


\section{DISCLAIMER}

This report was prepared as an account of work sponsored by an agency of the United States Government. Neither the United States Government nor any agency thereof, nor any of their employees, make any warranty, express or implied, or assumes any legal liability or responsibility for the accuracy, completeness, or usefulness of any information, apparatus, product, or process disclosed, or represents that its use would not infringe privately owned rights. Reference herein to any specific commercial. product, process, or service by trade name, trademark, manufacturer, or otherwise does not necessarily constitute or imply its endorsement, recommendation, or favoring by the United States Government or any agency thereof. The views and opinions of authors expressed herein do not necessarily state or reflect those of the United States Government or any agency thereof. 


\section{DISCLAIMER}

Portions of this document may be illegible in electronic image products. Images are produced from the best available original document. 


\title{
Whirl Plus Tilt
}

\author{
T. K. Fowler
}

\begin{abstract}
It is shown that, for an idealized rotor with identical magnetic bearings of negligible mass, precession and rotation are decoupled from the center-of-mass motion so that stabilization of whirl instabilities can be designed independent of tilt. The bearing torques that cause whirl also apply torques on the free-bodyrotational motion in a tilted state. The rotational equations of motion including these torques are given in the paper. An approximate solution for a special case suggests the possibility of tilt instability above a critical frequency.
\end{abstract}

\section{INTRODUCTION}

As before 1 , to calculate whirl we assume the bearings act at a point at each end of a rigid rotor shaft, labeled $A$ and $B$, and neglect spin torque on the shaft (constant spin $\omega$ ). The bearing forces have the form

$$
\overrightarrow{F_{i}}=\hat{x}\left\{-K_{i}\left(x_{i}-x_{l i}\right)+\alpha_{i}\left(y_{i}-y_{l i}\right)\right]+\hat{y}\left\{-K_{i}\left(y_{i}-y_{l i}\right)-\alpha_{i}\left(x_{i}-x_{l i}\right)\right\}
$$

where $\chi_{l i}, y_{l i}$ is the bearing center-of-force location for bearing $i=A$ or $B ; K_{i}$ is the effective spring constant; and $\alpha_{i}$ is a cross-coupling coefficient due to eddy current drag etc. 1 The force on the bearing due to its mounting is taken to be of the form

$$
\overrightarrow{F_{l i}}=-\hat{x} K_{x i} x_{l i}-\hat{y} K_{y i} y_{l i}
$$

where $K_{x}$ and $K_{y}$ are allowed to be different (e.g., a flexible mount clamped in the $\mathbf{x}$ direction to stiffen it in that direction). It has been shown that such an asymmetric mount stabilizes whirl. ${ }^{1}$ The addition of resistive damping in Eq. (2) would also be stabilizing. ${ }^{1,2}$

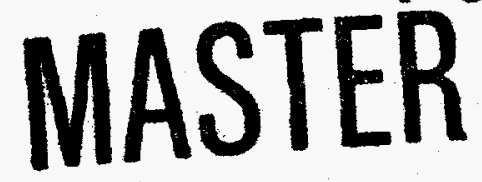


In Ref. 1, Appendix A, we considered tilt modes for cases in which bearing $A$ is laterally stabilizing $\left(K_{A}>0\right)$ but $B$ is destabilizing $\left(K_{B}<0\right.$; however, note that in that paper we defined $K_{B}$ positive and introduced the negative sign explicitly whereas here all $K^{\prime}$ 's are "with sign"). Having not found any interesting case of that nature, and having since concluded that eddy-currentstabilized bearings are promising, here we restrict ourselves to stable bearings only $\left(\mathrm{K}_{\mathrm{i}}>0\right)$ and focus on possible couplings of tilt and whirl not considered in Ref. 1.

For $K_{i}>0$, we can neglect $m \ddot{\overrightarrow{x_{i}}}$ for lightweight bearings ${ }^{1,2}$ and take the net force at each $\overrightarrow{x_{l i}}$ to be zero:

$$
\overrightarrow{F_{i i}}-\overrightarrow{F_{i}}=0
$$

This would be invalid if $K_{1}<0$ since $\overrightarrow{\mathbf{x}_{\mathbf{i}}}$ could become unbounded as the heavy rotor repels the lightweight bearing. Combining Eqs. (1) - (3) we can eliminate $\overrightarrow{x_{i}}$ to obtain

$$
\begin{aligned}
& \left.\vec{F}_{i}=\hat{x}\left\{-\dot{K}_{i x} x_{i}+\dot{\alpha}_{i} y_{i}\right\}+y i-\dot{K}_{i y} y_{i}-\dot{\alpha}_{i} y_{i}\right\} \\
& K_{i x}^{\prime}=\frac{K_{i} K_{x i}\left(K_{i}+K_{y i}\right)+\alpha_{i}^{2} K_{x i}}{\left(K_{i}+K_{x i}\right)\left(K_{i}+K_{y i}\right)+\alpha_{i}^{2}} \\
& K_{i y}^{\prime}=\frac{K_{i} K_{y i}\left(K_{i}+K_{x i}\right)+\alpha_{i}^{2} K_{y i}}{\left(K_{i}+K_{x i}\right)\left(K_{i}+K_{y i}\right)+\alpha_{i}^{2}} \\
& \alpha_{i}^{\prime}=\frac{\alpha_{i} K_{x i} K_{y i}}{\left(K_{i}+K_{x i}\right)\left(K_{i}+K_{y i}\right)+\alpha_{i}^{2}}
\end{aligned}
$$

In terms of the rotor Euler angles defined in Ref. 1, the shaft positions $A$ and $B$ are given by:

$$
\begin{aligned}
& x_{i}=x+\delta_{i} \sin \phi \\
& y_{i}=y-\delta_{i} \cos \phi
\end{aligned}
$$


where $\delta_{\mathrm{A}}=\ell_{\mathrm{A}} \sin \theta, \delta_{\mathrm{B}}=-\ell_{\mathrm{B}} \sin \theta$ and $\ell_{\mathrm{A}}, \ell_{\mathrm{B}}$ are the (positive) lengths along the shaft from the center of mass (C.M.) at $x, y$ to $\overrightarrow{x_{A}}$ and $\overrightarrow{x_{B}}$ at the ends of the shaft where the bearing forces act. Here $\phi$ is the angle of precession about a fixed $z$ axis and $\theta$ is the angle of tilt.

\section{SYMMETRIC ROTOR - TILT}

Before proceeding to the general equations of motion, we note that for the idealized case of a symmetric rotor $\left(\ell_{\mathrm{A}}=\ell_{\mathrm{B}}, \mathrm{K}_{\mathrm{A}}=\mathrm{K}_{\mathrm{B}}, \alpha_{\mathrm{A}}=\alpha_{\mathrm{B}}, \mathrm{K}_{\mathrm{yA}}=\mathrm{K}_{\mathrm{xB}}, \mathrm{K}_{\mathrm{yA}}\right.$ $=K_{y B}$ ), combining Eqs. (4) - (9) give $s$ as the combined force acting on the center of mass (C.M.):

$$
\vec{F}_{C . M .}=\vec{F}_{A^{+}} \overrightarrow{F_{B}}=\hat{x}\left\{-2 K_{x} x+2 \alpha^{\prime} y\right\}+\hat{y}\left\{-K_{y}^{\prime} y-2 \alpha^{\prime} x\right\}
$$

which is indepedent of the Euler angles. Hence, for the symmetric rotor of mass $M$, the whirl equation,

$$
M \ddot{\vec{x}}=\overrightarrow{F_{C}} \cdot M=\overrightarrow{F_{A}}+\overrightarrow{F_{B}}
$$

is independent of the Euler angles and there is no effect of tilting on the whirl. Substituting Eq. (10) into Eq. (11) for the symmetric rotor gives whirl instability if $K_{x^{\prime}}=K_{y^{\prime}}$ and stability if $\beta=K_{y^{\prime}} / K_{x}{ }^{\prime}$ is different from unity by a small amount depending on the magnitude of $\alpha^{\prime}$. For weak bearing forces $(\alpha, K x, y<K)$ this reduces to 1,2

$$
|1-\beta|>\frac{2 \alpha^{\prime}}{K_{x^{\prime}}} \cong \frac{2 \alpha K_{y}}{K^{2}}
$$

Asymmetry in any of the parameters (rotor not centered, bearing forces unequal), and also bearing inertia, would introduce Euler angle dependence and hence some back coupling of tilt on whirl. 


\section{EQUATIONS OF MOTION}

Even for the symmetric rotor, the fact that $\vec{F}_{A}$ and $\overrightarrow{F B}_{B}$ act at different locations can introduce torques in the rotational equations. The generalized torques (not limited to symmetry) are give by:

$$
\vec{N}_{i}=\left(\hat{z} \eta_{i} \ell_{i} \cos \theta+\hat{x} \delta_{i} \sin \phi-\hat{y} \delta_{i} \cos \phi\right) \times \vec{F}_{i}
$$

with $\eta_{\mathrm{A}}=1=-\eta_{\mathrm{B}}$ (recall $\ell_{\mathrm{A}}$ and $\ell_{\mathrm{B}}$ were taken positive). The general rotational equations of motion are ${ }^{1,3}$

$$
\begin{aligned}
& \frac{d}{d t} P_{\psi} \equiv \frac{d}{d t}\left(I_{1} \dot{\phi} \sin ^{2} \phi+P_{\psi} \cos \theta\right)=\hat{z} \cdot\left(\vec{N}_{A}+\vec{N}_{B}\right) \\
& \begin{array}{r}
\frac{d P_{\theta}}{d t} \equiv \frac{d}{d t}\left(I_{1} \dot{\theta}\right)=I_{1} \phi^{2} \sin \theta \cos \theta-\dot{\phi} P \psi \sin \theta \\
\quad+(\hat{x} \cos \phi+\hat{y} \sin \phi) \cdot\left(\vec{N}_{A}+\vec{N}_{B}\right)
\end{array} \\
& P_{\psi}=I_{3}(\dot{\psi}+\dot{\phi} \cos \theta)=I_{3} \omega
\end{aligned}
$$

where $\omega$ is taken constant. Note that these are Lagrangean equations, not the standard Euler equations about principal axes. Equation (14) is simply the equation for angular momentum about a fixed z-axis. Equation (15) is the Lagrangean equation for the tilt angle $\theta$ in which a torque of kinematic origin appears on the r.h.s. in addition to $\vec{N}_{A, B}$ both torques being taken about the socalled "line of nodes" rather than a principal axis. ${ }^{3}$ The conservation law Eq. (16) follows from neglecting spin torque as noted above (constant $\omega$ ), as is conventional in analyzing whirl. Additional terms would appear in the $\vec{N}_{i}$ if resistive damping is introduced in Eq. (2). Both $\alpha_{i}$ and resistive damping introduce non-conservative forces. Equations (14) - (16) are correct for any $\overrightarrow{\mathrm{F}}_{\mathrm{A}}$ and $\overrightarrow{\mathrm{F}}_{\mathrm{B}}$. Together with an equation for $\overrightarrow{\mathrm{x}}_{\mathbf{i}}$ when bearing inertia matters, Eqs. (11) and (14) - (16) completely describe the motion for all cases. 
The C.M. angular momentum equation derivable from Eq. (11), used in Ref. 1, is

$$
\frac{d}{d t} P_{\Omega}=\hat{z} \cdot \vec{R} \times\left(\vec{N}_{A}+\vec{N}_{B}\right)
$$

where $\overrightarrow{\mathbf{R}}=\hat{\mathrm{x}} \mathrm{x}+\hat{\mathrm{y}}, \mathrm{P}_{\Omega}=\mathrm{MR}^{2} \dot{\Omega}$ and $\Omega$ is the polar angle at $\mathrm{x}, \mathrm{y}$. For the case of conservative forces considered in Ref. 1, Appendix $A, L_{z}=P_{\Omega}+P_{\phi}$ is conserved but not in general. In particular, $L_{z}$ is not conserved with a bearing force of the form Eq. (1) and (2), with or without the addition of resistive damping in Eq. (2).

\section{SYMMETRIC ROTOR - PRECESSION}

Let us now again restrict attention to a symmetric rotor (C.M. centered, bearings identical) whereupon:

$$
\begin{aligned}
\vec{N}_{A}+\vec{N}_{B} & =\hat{x}\left\{-2 \delta \ell K_{y}^{\prime} \cos \theta \cos \phi+2 \ell \delta \alpha^{\prime} \cos \theta \sin \phi\right\} \\
& +\hat{y}\left\{-2 \delta \ell K_{x}^{\prime} \cos \theta \sin \phi-2 \ell \delta \alpha^{\prime} \cos \theta \cos \phi\right\} \\
& +\hat{z}\left\{-2 \delta^{2}\left(K_{x}^{\prime}+K_{y^{\prime}}\right) \sin \phi \cos \phi-2 \delta^{2} \alpha^{\prime}\right\}
\end{aligned}
$$

Combining Eqs. (10) - (18) we obtain as the complete set of equations for a symmetric rotor and weightless bearings:

$$
\begin{aligned}
& M \bar{z}=-2 K_{z} z \\
& M \bar{x}=-2 K_{x}^{\prime} x+2 \alpha^{\prime} y \\
& M \bar{y}=-2 K_{y}^{\prime} y-2 \alpha^{\prime} x \\
& P \psi=I_{3}(\dot{\psi}+\dot{\phi} \cos \theta)=I_{3} \omega\left(\frac{d P_{\psi}}{d t}=0\right)
\end{aligned}
$$




$$
\begin{aligned}
\frac{d P_{\varphi}}{d t}=\frac{d}{d t}\left(I_{1} \dot{\phi} \sin ^{2} \theta+P_{\psi} \cos \theta\right)=-2 \delta^{2}\left[\left(K_{x}{ }^{-}-K_{y^{\prime}}\right) \sin \phi \cos \phi+\alpha^{\prime}\right] \\
\begin{aligned}
\frac{d P_{\theta}}{d t}=\frac{d}{d t}\left(I_{1} \dot{\theta}\right) & =I_{1} \dot{\phi}^{2} \sin \theta \cos \theta-\dot{\phi} P_{\psi} \sin \theta \\
& -2 \delta \ell \cos \theta\left(K_{x} \sin ^{2} \phi+K_{y^{\prime}} \cos ^{2} \phi\right)
\end{aligned}
\end{aligned}
$$

where $\mathrm{K}_{\mathbf{z}}$ is the vertical spring constant and the other coefficients are given in Eqs. (5) - (7).

Thus we find that, for the idealized case of symmetry and weightless bearings, the rotational Eqs. (22) - (24) are independent of the C.M. coordinates, just as the C.M. Eqs. (19) - (21) are independent of the Euler angles. Hence tilt does not couple to whirl and whirl does not couple to tilt for the ideal case. Rather, a torque produced by bearing drag $\left(\alpha^{\prime} \neq 0\right)$ acts independently upon the C.M. and upon the rotor acting as a free object (no coupling to C.M.). This dragtorque causes whirl instability of the C.M. stabilized by $\mathrm{K}_{\mathrm{x}}^{\prime} \neq \mathrm{K}_{\mathrm{y}}^{\prime}$ which in turn produces an additional torque term in Eq. (23). Moreover an additional torque appears in Eq. (24) even with $\mathrm{K}_{\mathrm{x}}{ }^{\prime}=\mathrm{K}_{\mathrm{y}^{\prime}}$. These torques are proportional to the tilt $(\delta, \theta$ non-zero).

Except for the $\alpha^{\prime}$ term, these equations can be derived from a Lagrangean $\mathrm{L}=\mathrm{T}-\mathrm{V}$ with 1

$$
\begin{aligned}
& T=\frac{1}{2} I_{1}\left(\dot{\theta}^{2}+\dot{\phi}^{2} \sin ^{2} \theta\right)+\frac{1}{2} I_{3}(\dot{\psi}+\dot{\phi} \cos \theta)^{2} \\
& V=\frac{\delta^{2}}{2}\left(K_{x}^{\prime} \sin ^{2} \phi+K_{y}^{\prime} \cos ^{2} \phi\right)
\end{aligned}
$$

and corresponding energy

$$
\mathrm{H}=\mathrm{T}+\mathrm{V} \text {. }
$$


For $\alpha^{\prime}=0, H$ is a constant of the motion. Since all terms in $H$ are positive, we may conclude that the motion is stable $(\dot{\theta}, \dot{\phi}$ bounded by initial value of $\delta$ ) even for $\mathrm{K}_{\mathrm{x}}^{\prime} \neq \mathrm{K}_{\mathbf{y}^{\prime}}$. Including the $\alpha^{\prime}$ term gives by standard calculations

$$
\frac{\mathrm{dH}}{\mathrm{dt}}=-\dot{\phi} 2 \delta^{2} \alpha^{\prime}
$$

(in general $\dot{\mathrm{H}}=\Sigma \dot{\mathrm{q} F}$ for Lagrangean coordinates $\mathrm{q}$ with non-conservative forces $\mathrm{F}$ in $\left.\frac{\mathrm{d}}{\mathrm{dt}}\left(\frac{\partial \mathrm{L}}{\partial \dot{\mathrm{q}}}\right)=\frac{\partial \mathrm{L}}{2 \mathrm{q}}+\mathrm{F}\right) .^{3}$ Since we cannot be certain that $\dot{\phi}>0$ and therefore we cannot generate $\dot{\mathrm{H}} \leq 0$ from Eq. (28), it follows that we can no longer guarantee $\dot{\theta}$ and $\dot{\phi}$ are bounded by energy arguments alone.

Thus, for $\alpha^{\bullet} \neq 0$, we must examine rotational stability dynamically. Having assumed $P_{\psi}$ to be conserved, the motion is described by Eqs. (23) and (24) alone. These equations require numerical solution.

For small $\theta$, Eqs. (23) and (24) become:

$$
\begin{aligned}
& I_{1^{\prime}}\left(\ddot{\phi} \delta^{2}+\dot{\phi} \dot{\phi} \dot{\delta}\right)-P_{\psi^{\prime}} \delta \dot{\delta}=-2 \delta^{2}\left[\left(K_{x}^{\prime}-K_{y^{\prime}}\right) \sin \phi \cos \phi+\alpha^{\prime}\right] \\
& I_{1}{ }^{\prime}\left(\dot{\delta}-\dot{\phi}^{2} \delta\right)+\dot{\phi} P_{\varphi}^{\prime} \delta=-2 \delta\left[K_{x^{\prime}} \sin ^{2} \phi+K_{y^{\prime}} \cos ^{2} \phi\right]
\end{aligned}
$$

where $\delta \cong \ell \theta, \mathrm{I}_{1}{ }^{\prime}=\mathrm{I}_{1} / \ell^{2}, \mathrm{P}_{\psi^{\prime}}=\mathrm{P}_{\psi} / \ell^{2}$. These equations, being respectively quadratic and linear in $\delta$, are independent of the magnitude of the tilt angle $\theta$. The following approximate solution for a particular case suggests the possibility of instability above some frequency $\omega$. Let $\mathrm{K}_{\mathrm{x}}{ }^{\prime}=\mathrm{K}_{\mathrm{y}^{\prime}}$ (the whirl-unstable case) and let $\dot{\phi}$ equal a constant to be determined. Then, from Eq. (29),

$$
\delta \cong \delta_{0} \exp \frac{2 \alpha^{\prime}}{P_{\psi^{\prime}}} t
$$

if 


$$
P_{\psi^{\prime}}>>\left|2 I_{1^{\prime}} \dot{\phi}\right|
$$

which predicts unstable tilting at an exponential rate. The corresponding $\dot{\phi}$ found by substituting Eq. (31) into Eq. (30), still assuming Eq. (32), is (taking the smaller root)

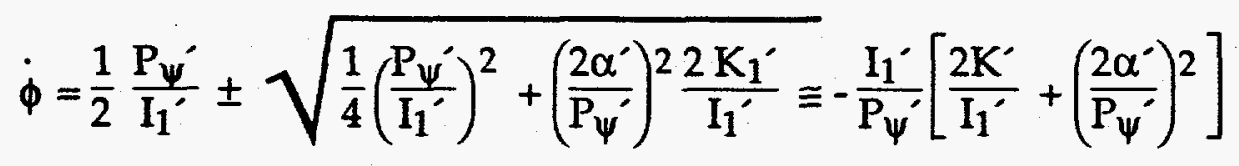

from which Eq. (32) becomes (for $\alpha^{\prime}<<K^{\prime}$ )

$$
P \psi^{\prime}>2 \sqrt{I_{1}^{\prime} K^{\prime}}
$$

Since $\mathrm{P} \psi=\mathrm{I}_{3} \omega$, Eq. (34) and hence Eq. (31) are valid only above some critical value of $\omega$. It will be interesting to determine, how $\mathrm{K}_{\mathrm{x}}{ }^{\prime} \neq \mathrm{K}_{\mathrm{y}^{\prime}}$, or adding damping in Eq. (2), would affect tilting.

Note that $\mathrm{K}_{\mathrm{y}}<<\mathrm{K}$, useful for whirl stabilization by Eq. (12), actually reduces the critical frequency for tilt by Eq. (34).

Note also that, since $\dot{\phi}<0$ by Eq. (33) for our special case of tilt instability, also $\dot{\mathrm{H}}>0$ for this case, by Eq. (28).

\section{SYMMETRIC ROTOR - DAMPING BEARINGS}

Damping in the bearings can be represented by an additional force term in Eq. (1) of the form

$$
\vec{F}_{\mathbf{i} \text { Damp }}=-v_{\mathbf{i}} \dot{\overrightarrow{\mathbf{x}_{i}}}
$$

Let us first consider very stiffly-mounted bearings $\left(K_{i x}, K_{i y} \gg K_{i}\right)$ in which case we can set $\vec{x}_{l i} \cong 0$ in Eq. (1). Again for the symmetric case there is no coupling of whirl and tilt, in which case Eq. (11) is independent of the Euler 
angles. Adding Eq. (35) into Eq. (11) then stabilizes the whirl if $v=v_{A}=v_{B}$ is large enough. Solving the quadratic eigenvalue equation for the symmetric case gives as the criterion for stability, 4

$$
v>\alpha\left(\frac{\mathrm{K}}{\mathrm{M}}\right)^{-1 / 2}
$$

The eddy currents that give rise to $\alpha$ and $\mathrm{K}$ in eddy-current stabilized magnetic bearings also give $v \neq 0$, and indeed some damping in the bearings is desirable in vehicular applications. 1 Borrowing from Ref. 1, Eq. (E-13) with the addition of Eq. (35) becomes

$$
\begin{aligned}
& M \ddot{x}=-K x+\alpha y-v \dot{x} \\
& M \ddot{y}=-K y-\alpha y-v \dot{y}
\end{aligned}
$$

when

$$
\begin{aligned}
& \alpha=K \frac{\delta}{\mathrm{a}} \frac{\overline{x^{2}}}{\Delta^{2}} \\
& v=\frac{\omega_{0}}{\omega^{2}} \frac{\delta}{\Delta} \mathrm{K}
\end{aligned}
$$

Here $\delta$ is the frequency-dependent skin depth, $\Delta$ is the gap spacing (see Fig. 5 , Ref. 1), $a$ is the bearing radius, $K$ is its effective spring constant and $\omega_{0}=$ $(K / M)^{1 / 2}$. Eq. (39) is taken from Ref. 1 with $x^{3} \cong \overline{x^{2}} x$, typically $x / \Delta \cong 0.1$ being the range of interest. Eq. (40) is taken from Eqs. (35) - (37) in Ref. 1, where it is estimated that the damping power is a factor $\omega_{0}^{2} / \omega^{2}$ smaller than the rotational power loss giving rise to $\alpha$ and using

$$
P_{\text {Damp }} \equiv(x \omega) \cdot\left(v \times \omega_{0}\right)
$$

Substituting Eqs. (39) and (40) into Eq. (36) gives the stability criterion. 


$$
\omega_{\min }<\omega<\omega_{0}\left[\frac{a}{\Delta}\left(\frac{x^{2}}{\Delta^{2}}\right)^{-1}\right]^{1 / 2}
$$

where $\omega_{\min }$ is a lower bound to assure $\delta<\Delta$ which is the condition that $K$ be usefully large for this case.

This criterion, only crudley derived here, would appear to place stringent conditions on stability. Moreover, further study is needed to determine tilt stability with the additional rotational torque produced by Eq. (35).

\section{SYMMETRIC ROTOR - DAMPING IN THE BEARING MOUNTS}

Damping in the bearing mounts can be represented by an additional force term in Eq. (2) of the form

$$
\vec{F}_{1 i}=-v_{1 i} \vec{x}_{1 i}
$$

With this addition, it is no longer possible exactly to eliminate $\overrightarrow{x_{1}}$ for weightless bearings, but we can do so approximately if we treat the $v_{i}$ and $v_{1 i}$ terms as perturbations and drop these terms in finding $\overrightarrow{x_{1}}$ by solving Eq. (3). The resulting equations are very complicated but again, for the symmetric case, tilt and whirl decouple and the problem reduces to solving Eq. (11) for whirl and solving Eqs. (23) and (24) for tilt with the appropriate damping forces added. As in our earlier examples, the decoupling of tilt and whirl follows because $F_{A}$ and $F_{B}$ are linear combinations of $x, x y$ and $y$. Then, using Eqs. (8) and (9), for the symmetric case combinations $x_{A}+x_{B}$ or $\dot{x_{A^{+}}}+\dot{x_{B}}$ eliminate Euler angles in Eq. (11) and combinations $x_{A}-x_{B}$ and $\dot{x}_{A^{-}} \dot{x_{B}}$ eliminate C.M. coordinates in the rotational equations.

To simplify the algebra, we now consider not stiff bearings but very "floppy" bearings with $\mathrm{K}_{\mathrm{x}}, \mathrm{K}_{\mathrm{y}}, \alpha<\mathrm{K} .1,2$ Then, carrying out the approximate elimination of $\overrightarrow{x_{1}}$ described above gives 


$$
\begin{aligned}
F_{i} & =\hat{x}\left\{-K_{x i} x_{i}+\alpha_{i} y_{i}-v_{1 i} \dot{x}_{i}\right\} \\
& +\hat{y}\left\{-K_{y i} y_{i}-\alpha_{i}{ }^{\prime} x_{i}-v_{1 i} \dot{y}_{i}\right\}
\end{aligned}
$$

That is, the system behaves as if the bearing forces are replaced by those of the bearing mounts (Eq. (1) and (43)) except that there is a residual torque $\alpha^{\prime}$ of order $\alpha\left(K_{x} K_{y} / K^{2}\right)$ by Eq. (7) for the "floppy" case. Then to lowest order neither the bearing spring constants $K_{i}$ nor the bearing damping $v_{i}$ enter directly in the force $\mathbf{F}_{\mathbf{i}}$.

Introducing Eq. (44) with Eqs. (8) and (9) into Eq. (11) eliminates the Euler angles for the symmetric case, to give the whirl equation,

$$
M \ddot{\vec{x}}=\hat{x}\left\{-2 K_{x} x+2 \alpha^{\prime} y-2 v_{1} \dot{x}\right\}+\hat{y}\left\{-2 K_{y} y-2 \alpha^{\prime} x-2 v_{1} \dot{y}\right\}
$$

The eigenvalue equation is now quartic. Stability criteria, derived in Ref. 1 Appendix E, are given by Eq. (12) for $v_{1}=0$ and, for $v_{1} \neq 0$ but $K_{x}=K_{y}$, by

$$
\alpha<v_{1}\left(\frac{K}{M}\right)^{1 / 2}\left(\frac{K}{K_{x}}\right)^{3 / 2}
$$

The tilt equations for this case, still assuming symmetry, are given by Eqs. (23) and (24) with additional torque terms given by, for Eq. (23),

$$
\left(\vec{N}_{A^{+}} \vec{N}_{B}\right)_{z} \text { Damp }=-2 v_{1} \delta^{2} \dot{\phi}
$$

and, for Eq. (24),

$$
\left(\vec{N}_{A^{+}} \vec{N}_{B}\right)_{\perp \text { Damp }}=-2 v_{1} \ell \cos \theta \dot{\delta}
$$


For small $\theta$, Eqs. (29) and (30) become

$$
\begin{aligned}
& \left.\mathrm{I}_{1} \cdot \ddot{\phi} \delta^{2}+2 \phi \delta \dot{\delta}\right)-\mathrm{P}_{\psi}^{\prime} \delta \dot{\delta}= \\
& -2 \delta^{2}\left[\left(K_{x}-K_{y}\right) \sin \phi \cos \phi+\alpha^{\prime}\right) \\
& -2 v_{1} \delta^{2} \dot{\phi} \\
& I_{1}^{\prime}\left(\ddot{\delta}-\dot{\phi}^{2} \delta\right)+\dot{\phi} P_{\psi}^{\prime} \delta=-2 \delta\left[K_{x} \sin ^{2} \phi+K_{y} \cos ^{2} \phi\right]-2 v_{1} \dot{\delta}
\end{aligned}
$$

The special case of Sec. 4 again follows with constant $\phi$ given by Eq. (33) for small $v_{1}$, but Eq. (31) is now replaced by

$$
\delta \cong \delta_{0} \exp \frac{2\left(\alpha^{\prime}+v_{1} \dot{\phi}\right)}{P \psi^{\prime}} t
$$

Since $\dot{\phi}$ is negative, stability follows if $\left|v_{1}, \dot{\phi}\right|>\alpha^{\prime}$ or, using $\alpha^{\prime} \simeq K_{x}^{2} / K^{2}$ for floppy bearings and dropping small $a^{\prime}$ terms in $\dot{\phi}$, stability follows if, approximately,

$$
\alpha<v_{1}\left(\frac{2 \omega_{0}^{4}}{m_{3}{\omega \omega_{1}^{2}}^{2}}\right) \quad \text { TILT }
$$

where $\mathrm{m}_{3}=\mathrm{I}_{3} / M \ell^{2}, \omega_{0}^{2}=K / M$ and $\omega_{1}^{2}=K_{x} / M$. This criterion for "floppy" bearings should be much more easily satisfied than criterion Eq. (36) for stiffly mounted bearings. Again, here $M$ is the rotor mass, $I_{3}$ is its moment of inertia about the axis, $K$ is the magnetic bearing spring constant, and $K_{x}$ is the spring constant for the bearing mounting.

Criterion Eq. (52) for tilt stability for floppy bearings can be compared to the criterion for damping stabilization of whirl. For floppy bearings the whirl stabilization criterion, from Ref. 2 or Eq. (E-6) of Ref. 1, is 


$$
\alpha<v_{1}\left(\frac{\omega_{0}^{4}}{\omega_{1}^{3}}\right) . \quad \text { WHIRL }
$$

Comparing Eqs. (52) and (53), we see that the tilt stabilization criterion is the more difficult to satisfy, by a factor $\left(2 \omega_{1} / \omega \mathrm{m}_{3}\right)$.

Thus, it appears that tilt rather than whirl determines the critical frequency below which the system is stabilized by damping. The criteria for stabilization by anisotropy $\left(\mathrm{K}_{\mathrm{x}} \neq \mathrm{K}_{\mathrm{y}}\right)$ requires numerical solution.

Finally, we note that the "pendulum effect" in mechanical bearing systems is of no effect here. The pendulum effect applies to a rotor suspended from a mechanical bearing. Then any tilting of the rotor shaft necessarily lifts the rotor mass a distance

$$
\delta z=\frac{1}{2} \ell \theta^{2}
$$

where $\ell$ is the lever arm. Since the gravitational potential is $\mathrm{Mg} \delta \mathrm{z}$, this second order energy introduces a term in the $\theta$-equation that is linear in $\theta$. However, for magnetic bearings, tilting can occur while the center of mass remains fixed. Then there is no gravitational rotoring force. There is a small change of energy

$$
\delta \mathrm{V}=\frac{1}{2} \mathrm{Kz} \delta \mathrm{z}^{2}
$$

due to the spring constant, but this energy is fourth order in $\theta$ and produces a term a $\theta^{3}$ in the $\theta$-equation. That tilting can occur with fixed center of mass has already been demonstrated for the symmetric rotor. It is also true for a rotor suspended from a single magnetic bearing since the rotor shaft is "attached" only via the bearing effective spring constants. 


\section{References}

1. D. E. Baldwin, T. K. Fowler and R. F. Post, "Status of Passive Magnetic Bearing Designs," UCRL-ID-114231, July 21, 1993, Controlled Distribution.

2. E. J. Gunter, Jr., "Dynamics and Stability of Rotor-Bearing Systems," NASA SP-113 (1966).

3. H. Goldstein, Classical Mechanics, Chapters 4 and 5, Addison-Wesley, New York, 1953. 